\title{
PRODUCCIÓN DE BIODIESEL \\ A PARTIR DE ACEITE DE COCINA USADO
}

Mercedes Vera Mahecha

Felipe Vargas Torres

Juan Sebastián Buitrago

\section{Universidad de La Salle - Semillero EMTROPIQ}

\section{Resumen:}

Los biocombustibles son fuentes de energía renovable que reducen el impacto ambiental de los combustibles tradicionales y a su vez reducen la generación de gases de efecto invernadero. Se estima que el $75 \%$ del costo final del biodiésel corresponde a las materias primas, por lo cual la consecución de estas representa una de las principales limitaciones en el proceso. Por estos motivos el aceite de cocina usado se propone como una fuente de materia prima.

El aceite usado de cocina tiene un alto valor ácido, lo cual representa un desafío adicional para la producción de biodiésel, ya que esta característica promueve la generación de jabones y reduce la conversión y rendimiento. Para superar esta limitación se ha propuesto la implementación de una etapa primaria de esterificación ácida. Sin embargo, pocos estudios revelan las condiciones de operación de este proceso.

El propósito de este trabajo fue estudiar la producción de biodiesel a partir de aceite usado de cocina. El aceite fue obtenido de un restaurante de hamburguesas. Inicialmente, se realizó una filtración del aceite para la remoción de sólidos suspendidos, el contenido original de ácidos grasos libres del aceite fue de $4,73 \mathrm{mg} \mathrm{KOH/gr}$ y el índice de peróxidos fue de 4,74\% por lo cual, la etapa primaria de esterificación ácida fue requerida. Posteriormente, se desarrolló la neutralización del catalizador ácido y la etapa secundaria de transesterificación en medio básico. Finalmente, el biodiésel se separó, purificó y fueron desarrolladas pruebas fisicoquímicas para verificar su calidad, obteniendo que tanto su valor ácido como su viscosidad disminuye teniendo un valor de 0,21 mg KOH/gr y 29,26 mPa*s respectivamente. 


\section{Pallabras claves:}

aceite; biodiésel; caracterización del aceite; ácidos grasos libres; esterificación ácida.

\section{Abstract:}

The biofuels are renewable energy sources that reduce the environmental impact of the traditional fuels and at the same time reduce the generation of greenhouse gases. It is estimated that the $75 \%$ of the final cost of biodiesel corresponds to the raw materials, whereby the achievement of this represent one of the main limitations in the process. For these reasons the waste cooking oil is proposed like a sources of raw material.

The waste cooking oil has a high acid value, which represents a additional challenge for the biodiesel production, as this characteristic promotes the soap generation and reduce the conversion and performance. To overcome this limitation has been proposed the implementation of a primary stage of acid esterification. However, few studies reveal the conditions of operation of this process.

The purpose of this work was study the biodiesel production from waste cooking oil. The oil was obtained from a hamburger restaurant. Initially, it was done a oil filtration for the removal of suspended solids, the original content of free fatty acids of the oil was 4,73 $\mathrm{mg} \mathrm{KOH} / \mathrm{gr}$ and the peroxide index was 4,74\% whereby, the primary stage of acid esterification was required. Later, it was developed the neutralization of the acid catalyst and the secondary stage of transesterification in basic medium. Finally, the biodiesel was separated, purified and was developed physicochemical tests to verificate its quality, getting that its acid value and the viscosity decreasing having a value of 0,21 mg KOH/gr y 29,26 mPa*s respectively.

\section{Keywords:}

oil; biodiesel; oil characterization; free fatty acids; acid esterification. 


\section{Introducción}

A través de los tiempos, el consumo de combustibles fósiles, a nivel mundial ha aumentado notablemente debido a las necesidades del hombre; el consumo excesivo de estos productos ha causado efectos contraproducentes desde el punto de vista económico, social y ambiental, esto nos lleva a buscar nuevas alternativas más sustentables y más amigables con el medio ambiente. El biodiesel, como combustible alternativo, tiene muchos méritos. Se deriva de un recurso renovable, lo que alivia la dependencia de las importaciones de combustible de petróleo. Es biodegradable y no tóxico. En comparación con el diésel a base de petróleo, el biodiesel tiene un perfil de emisión de combustión más favorable, como bajas emisiones de monóxido de carbono, materia particular e hidrocarburos sin quemar [1].

El biodiesel se puede obtener a través de diferentes procesos, siendo la transesterificación el método más utilizado debido a su simplicidad técnica y eficiencia. La transesterificación alcalina catalizada del aceite de cocina residual es algo limitada en presencia de altas concentraciones de ácidos grasos libres y agua, dado que favorece la reacción de saponificación [2].

La Agencia Internacional de Energía predice que los biocombustibles proporcionarán aproximadamente el $27 \%$ del combustible de transporte mundial para 2050, pero actualmente los biocombustibles sólo proporcionan el $2 \%$ del combustible de transporte total [3].

Según el CONPES 3510 del 2008, el cual es un lineamiento de política para promover la producción sostenible de biocombustibles en Colombia, la importancia del biodiesel se basa en estos tres pilares [4]: 
Figura 1.

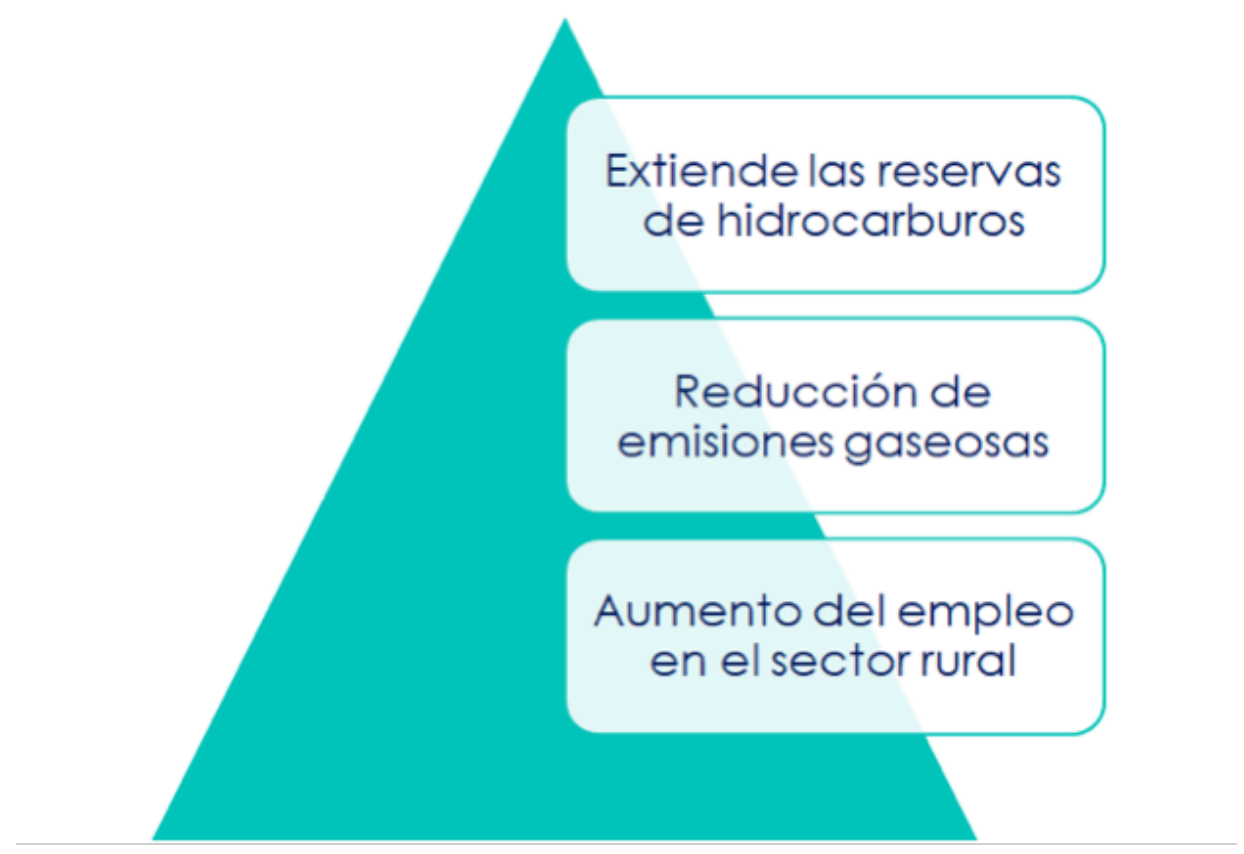

Fuente: elaboración propia.

La dirección de Fedebiocombustibles sostuvo que la meta es llegar a una mezcla del 15\% tanto en etanol como en biodiesel al año 2015 y del 20\% al 2020 para lo cual se requieren inversiones importantes [5].

En la figura 1 podemos observar la demanda nacional de biodiesel en Colombia. En ella desde el año 2018 hasta el presente se tiene una demanda constante de biodiesel, teniendo un promedio de aproximadamente 50.000 toneladas por mes. 
Figura 2. Demanda nacional de biodiesel

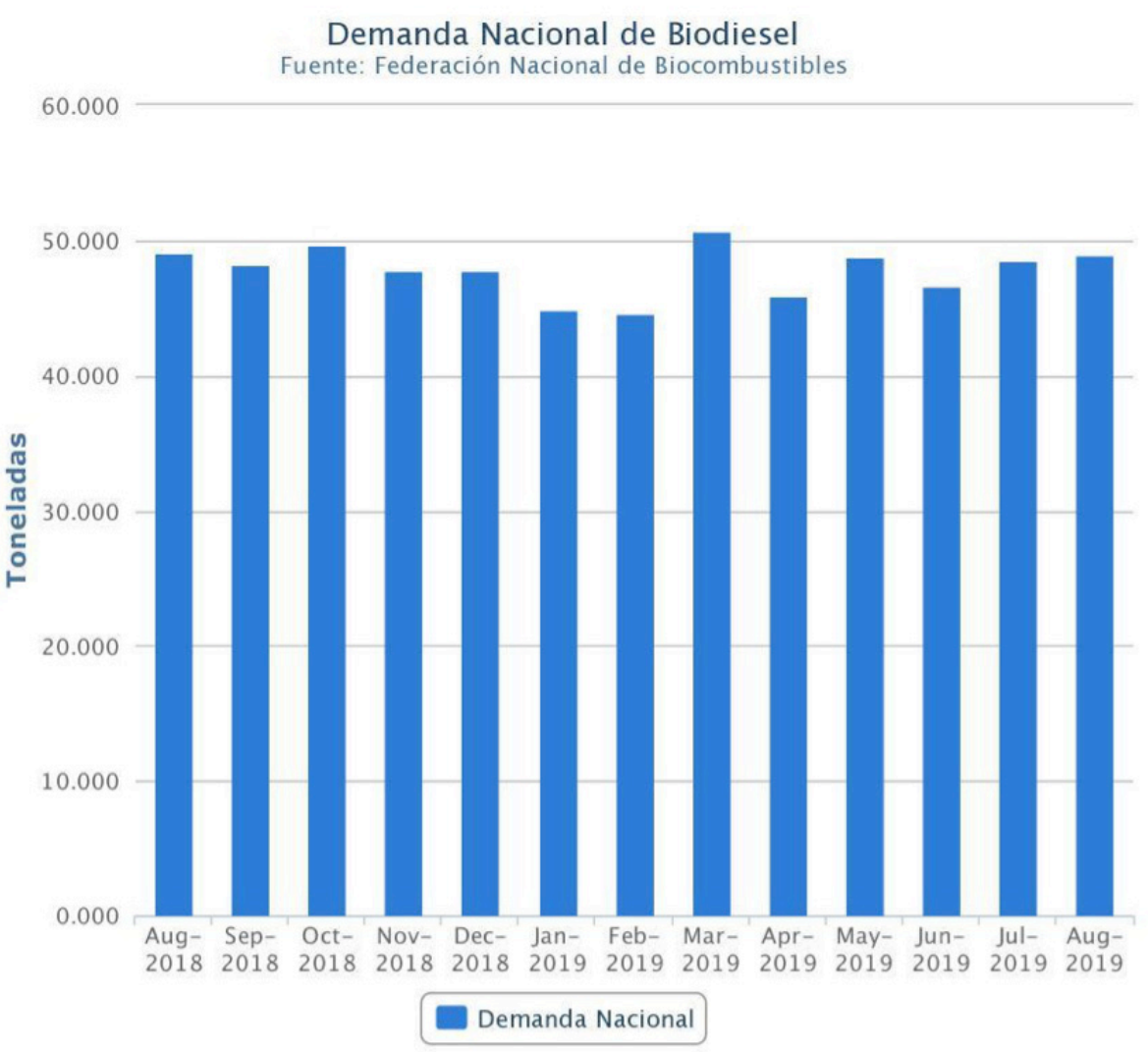

En la figura 2, podemos observar el crecimiento de la producción de aceite en diferentes departamentos de Colombia, desde el año 2014 hasta 2016. Esta producción ha ido aumentando, se tiene en cuenta que este es un beneficio para la producción de biodiesel a partir de aceite de cocina usado debido a que el objetivo es tomar el 5\% de la producción bruta de aceite en Bogotá, ya que en su gran mayoría este terminará siendo un desecho. 
Figura 3. Producción de aceite en Colombia

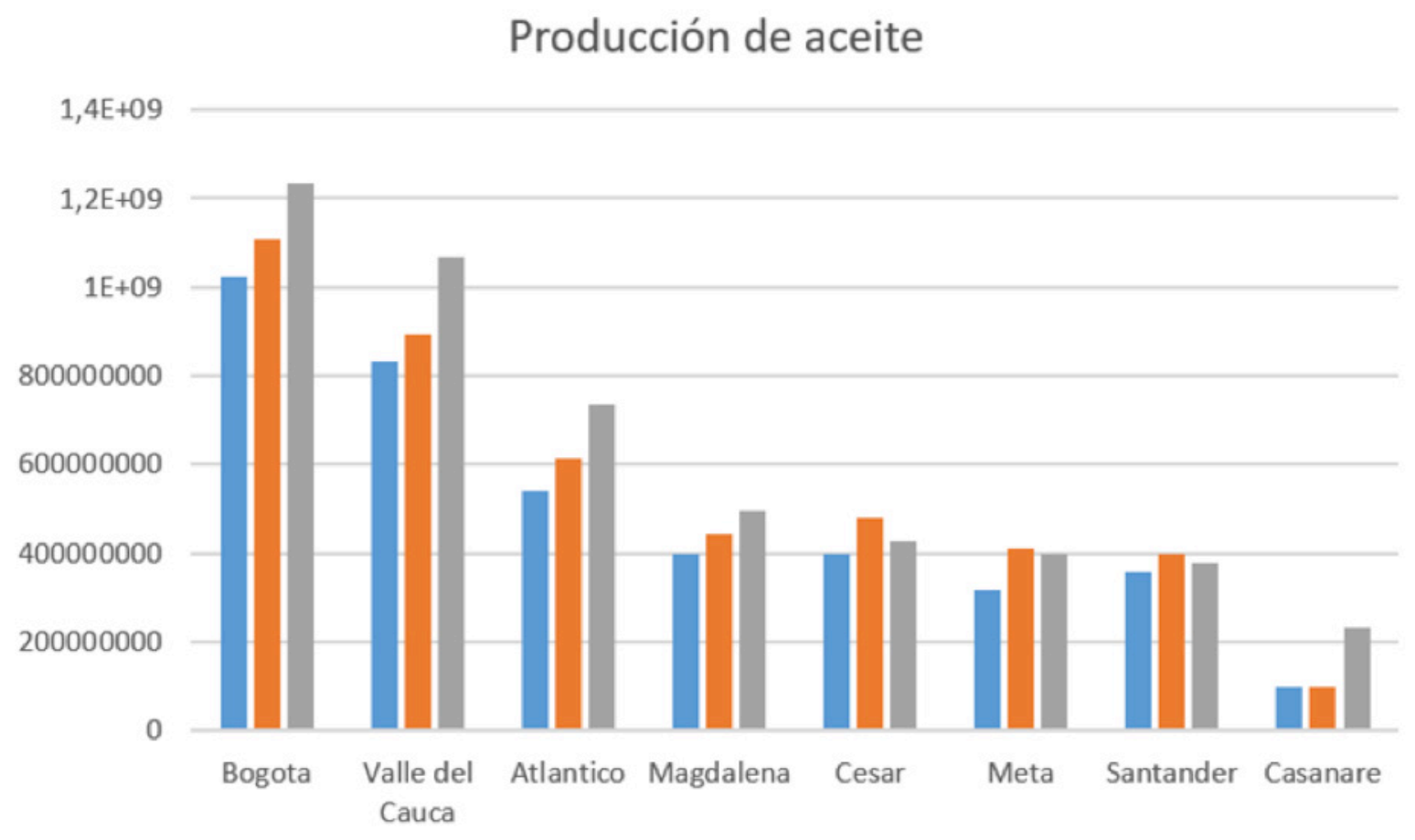

— Produccion bruta 2014 (USD) - Produccion bruta 2015 (USD) = Produccion bruta 2016 (USD)

Fuente: Encuesta Anual Manufacturera 2014-2015-2016.

En Colombia el aceite usado de cocina generalmente es desechado. Por ello, en este proyecto se busca aprovecharlo como una materia prima para la obtención de biodiesel. Para esto, se realizó una caracterización, un pretratamiento (esterificación ácida) y finalmente se obtuvo el biodiesel por medio de una transesterificación básica [6]. 
El consumo de aceite por restaurante es de 8439 litros anuales de los cuales 107 puntos están ubicados en la ciudad de Bogotá dando como resultado un consumo anual de 238.504 galones anuales [7].

El objetivo principal de este proyecto es obtener biodiésel a partir del aceite de cocina usado, para lo cual se implementarán unas etapas fundamentales que genere buen rendimiento del proceso. Inicialmente, se requiere realizar la caracterización fisicoquímica del aceite de cocina usado para determinar las condiciones que se tendrán en cuanta durante el proceso de producción de biodiésel; por consiguiente, se realiza la neutralización del aceite mediante una reacción de catálisis ácida con ácido sulfúrico. Teniendo este aceite con un menor valor ácido, se obtiene biodiesel mediante una transesterificación alcalina. Por último, para evaluar si el biodiesel producido puede ser llevado al mercado, se realiza una caracterización fisicoquímica de este.

\section{Materiales y métodos}

Inicialmente, se realizó una filtración al vacío del aceite, así para la caracterización del mismo se basó en cuatro pruebas, dos de ellas (índice de acidez y peróxidos) se realizaron según las normas NTC 218 y 236, respectivamente, y las otras dos (viscosidad y densidad) se realizaron por el método de picnómetros y con el viscosímetro Brookfield.

La reacción del proceso se divide en dos etapas: la primera, corresponde a la esterificación del aceite la cual se realizó con el fin de neutralizar la acidez del mismo y la transesterificación que es donde se obtiene el biodiesel. Ya que el biodiesel obtenido presenta impurezas, se realiza un lavado con agua destilada y finalmente se realiza el secado en una mufla a $105^{\circ} \mathrm{C}$. En la tabla 1 se muestra que materiales se usaron en cada uno de los métodos. 
Figura 4.

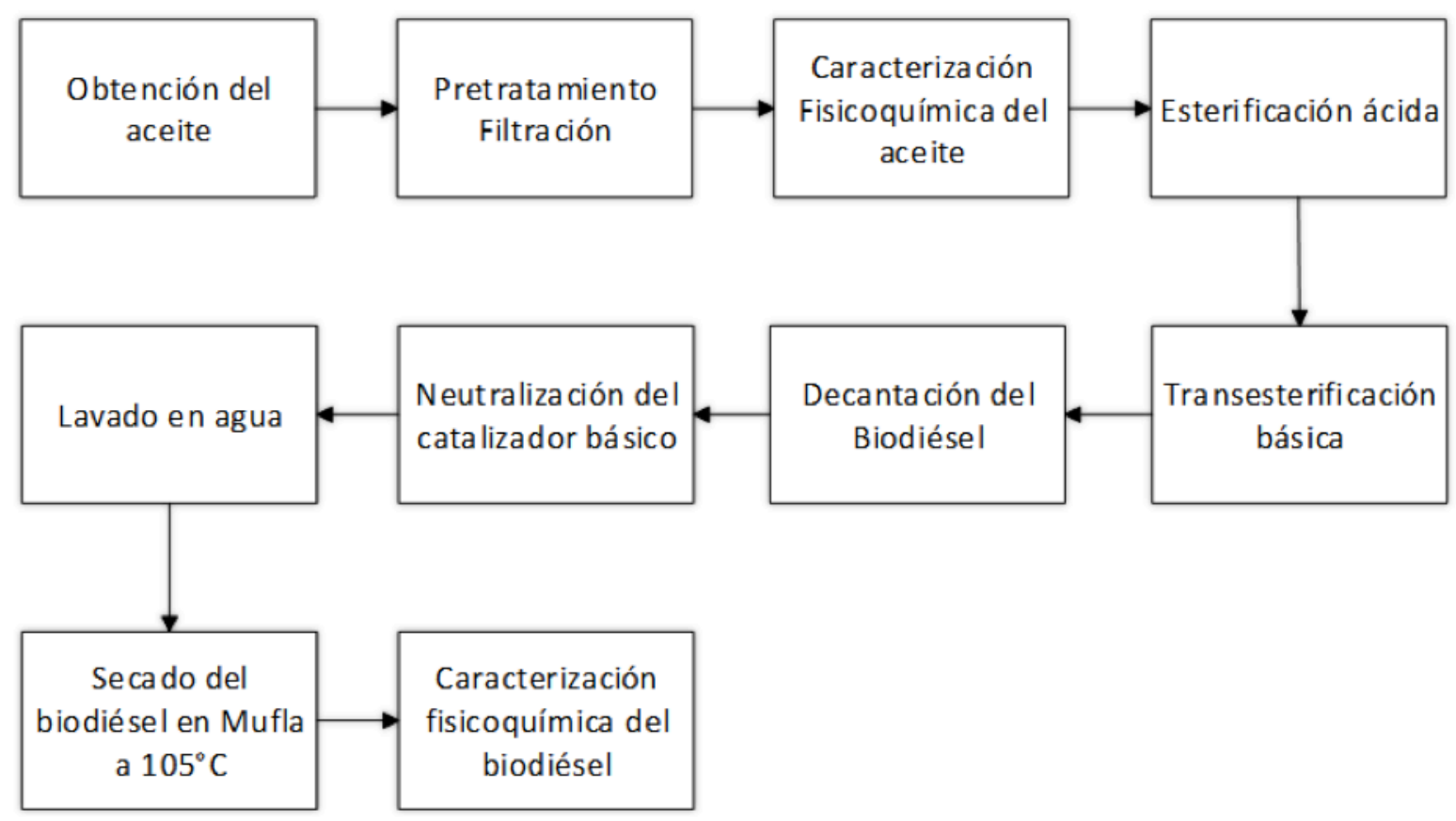

Fuente: Elaboración propia

Tabla 1. Método y materiales usados

\begin{tabular}{|l|l|}
\hline Método & Materiales \\
\hline Filtración & Bomba \\
& Manguera \\
& Trampa de vacío \\
& Embudo Buchner \\
& Papel filtro \\
\hline
\end{tabular}




\begin{tabular}{|c|c|}
\hline Densidad & \begin{tabular}{|l} 
Picnómetro \\
Pipeta \\
Pipeteador \\
Balanza
\end{tabular} \\
\hline Peróxidos & $\begin{array}{l}\text { Probeta } \\
\text { Erlenmeyer } \\
\text { Ácido acético } \\
\text { Cloroformo } \\
\text { Bureta } \\
\text { Soporte universal } \\
\text { Solución de almidón } \\
\text { Tiosulfato de sodio } \\
\text { Yoduro de Potasio }\end{array}$ \\
\hline Acidez & $\begin{array}{l}\text { Erlenmeyer } \\
\text { Mezcla alcohol-éter } \\
\text { Fenolftaleína } \\
\text { Hidróxido de potasio }\end{array}$ \\
\hline Viscosidad & Viscosímetro DV-2 \\
\hline Esterificación & $\begin{array}{l}\text { Ácido sulfúrico } \\
\text { Metanol } \\
\text { Balón de fondo plano } \\
\text { Agitador magnético } \\
\text { Condensador } \\
\text { Mangueras } \\
\text { Plancha de calentamiento }\end{array}$ \\
\hline
\end{tabular}




\begin{tabular}{|l|l|}
\hline Transesterificación & Hidróxido de sodio \\
& Metanol \\
& Balón de fondo plano \\
& Agitador magnético \\
& Condensador \\
& Mangueras \\
& Plancha de calentamiento \\
\hline Lavado & Agua destilada \\
& Plancha de calentamiento \\
& Agitador magnético \\
& Embudo de decantación \\
\hline Secado & Erlenmeyer \\
\hline
\end{tabular}

Fuente: Elaboración propia

\section{Resultados y discusión}

\section{Pretratamiento del aceite.}

\section{Obtención de la muestra.}

La muestra de aproximadamente $600 \mathrm{ml}$ de aceite a utilizar se obtuvo en un restaurante ubicado en la ciudad de Bogotá. Este presenta algunos sólidos suspendidos, es de color negro, homogéneo y poco viscoso. 
Figura 5. Muestra obtenida

\section{Filtración.}

Para eliminar las impurezas del aceite usado de cocina y los sólidos suspendidos en él, se realiza un proceso de filtración al vacío usando papel filtro, previamente se calienta el aceite a una temperatura de aproximadamente $50{ }^{\circ} \mathrm{C}$ para facilitar la filtración.

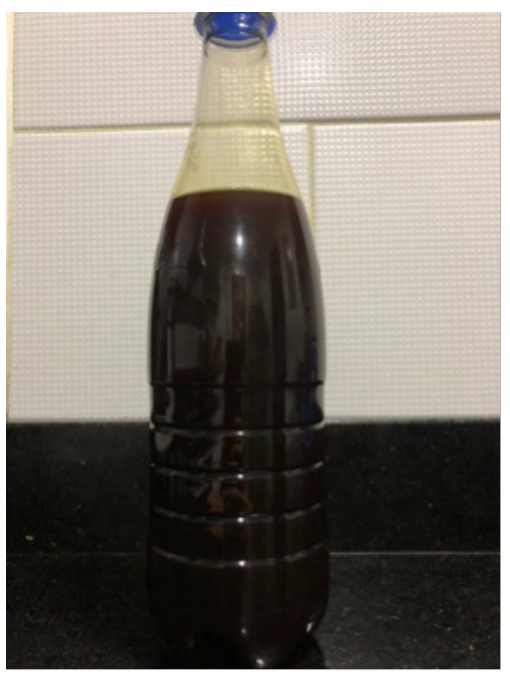

Figura 5. Montaje filtración al vacío

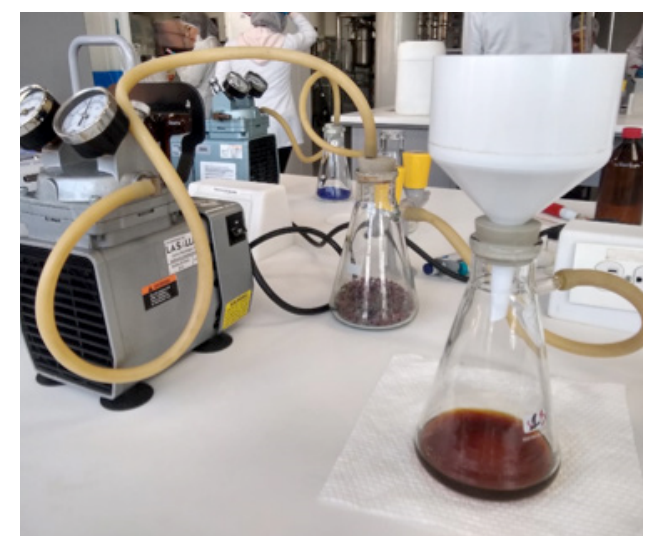

Figura 6. Papeles filtro

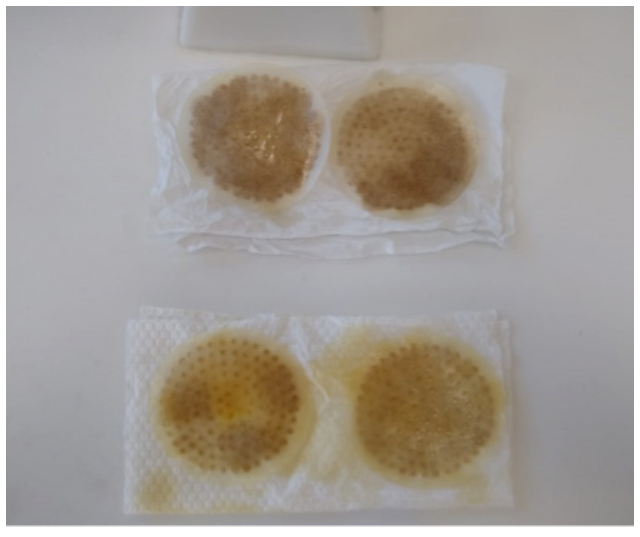

Figura 7. Peso final obtenido

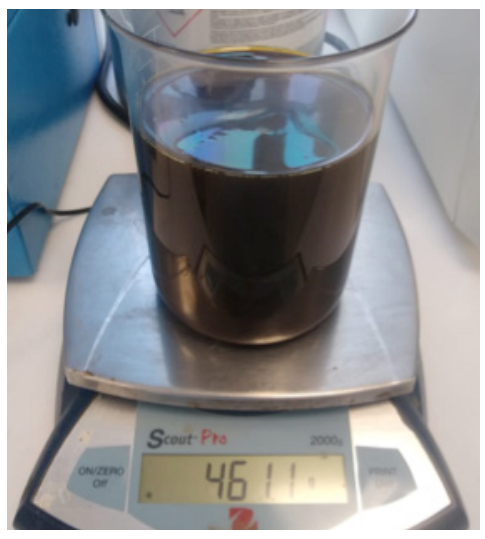

El proceso de filtrado mostrado en la figura 5 tardó aproximadamente 40 minutos, por otro lado, en la figura 6 se muestran los cuatro papeles filtro con los residuos del aceite, donde se observa que a medida que se iba realizando la filtración las impurezas retenidas en los papeles filtro iban disminuyendo. Finalmente se obtuvo una masa final del aceite de 461,1 g, como se puede apreciar en la figura 7. 
En la tabla 3 se muestran la masa de residuos obtenida en los papeles filtro:

Tabla 3. Peso de los residuos del aceite

\begin{tabular}{|c|c|c|c|}
\hline Papel filtro & $\begin{array}{l}\text { Peso inicial filtro } \\
\text { (g) }\end{array}$ & $\begin{array}{l}\text { Peso final filtro } \\
\text { (g) }\end{array}$ & Peso obtenido \\
\hline 1 & 0,4178 & 0,8788 & 0,461 \\
\hline 2 & 0,4103 & 0,8668 & 0,4565 \\
\hline 3 & 0,415 & 0,8589 & 0,4439 \\
\hline 4 & 0,4167 & 0,8298 & 0,4131 \\
\hline & & TOTAL & 1,7745 \\
\hline
\end{tabular}

Caracterización del aceite.

Densidad: Como se muestra en la imagen 2.1, se utilizaron 2 picnómetros de 25 mL cada uno. Para el cálculo de la densidad, se utilizó la siguiente expresión:

Figura 8. Picnómetros con el aceite

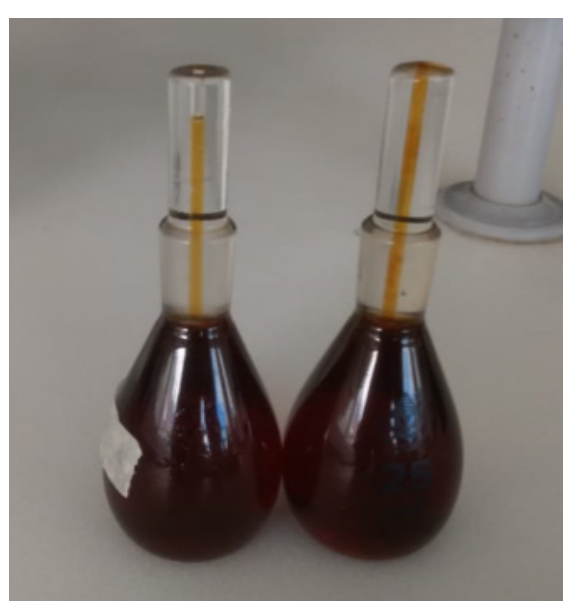




$$
\rho=\frac{m}{v}
$$

Donde:

p: Densidad.

m: Masa.

v: Volumen.

En la tabla 4 se muestran los resultados obtenidos para la densidad del aceite.

Tabla 4. Resultados de la densidad del aceite

\begin{tabular}{|rr|r|r|r|r|}
\hline Picnómetro & $\begin{array}{l}\text { Peso } \\
\text { (g) }\end{array}$ & picnómetro & $\begin{array}{l}\text { Peso con el aceite } \\
\text { (g) }\end{array}$ & Masa del aceite $(\mathrm{g})$ & Densidad (g/ml) \\
\hline 1 & 24,5932 & 47,5935 & 23,0003 & 0,920012 \\
\hline 2 & 25,0853 & 47,9961 & 22,9108 & 0,916432 \\
\hline
\end{tabular}

Viscosidad: Como se muestra en la figura 9 se utilizó el viscosímetro Brookfield, basado en el principio de viscosimetría rotacional. 
Figura 9. Viscosímetro Brookfield

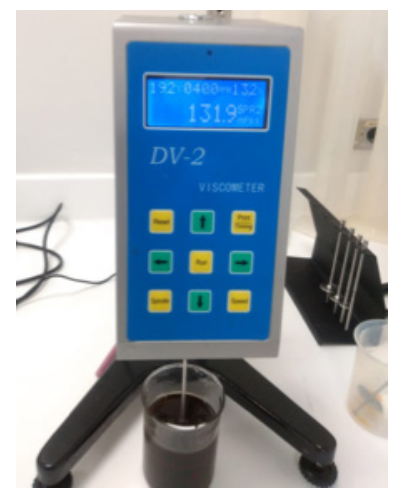

Se obtuvo una viscosidad de 131,9 mPa*s a temperatura ambiente de $19,2^{\circ} \mathrm{C}$ Índice de ácidos grasos libres: El proceso de la determinación de ácidos grasos libres se realizó según la norma NTC 218, en la figura 10 se muestra la titulación con el hidróxido de sodio 0,1 N y en la figura 11 se observa el cambio de color que se obtuvo.

Figura 9. Titulación-Determinación de acidez

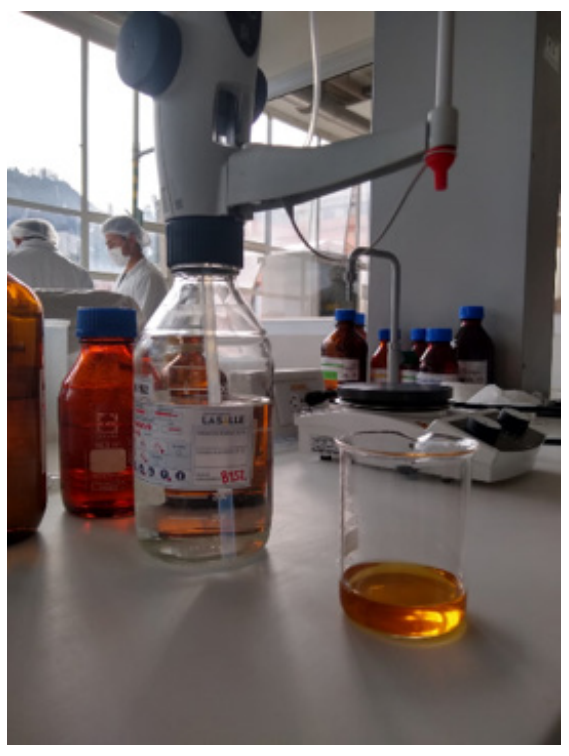

Figura 10. Cambio de color obtenido

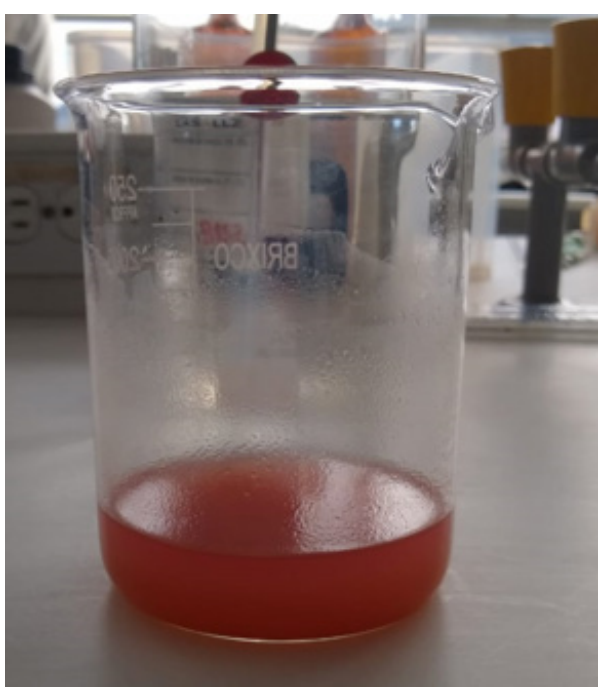


El \% de ácidos grasos libres o grado de acidez se determinó por la ecuación:

Ácidos grasos libres $=\underline{(5,61 * V * N * 100)}$ $P$

Donde:

$V=$ volumen $(\mathrm{ml})$ de $\mathrm{NaOH}$ consumidos

$\mathrm{N}=$ Normalidad del $\mathrm{NaOH}$

$\mathrm{P}=$ peso $(\mathrm{g})$ de aceite

Después de obtener el grado de acidez se procede a calcular el índice de acidez, dado por la siguiente ecuación:

Ácidos grasos libres $=(5,61 * 4,31 \mathrm{~mL} * 0,1 \mathrm{~g} / \mathrm{mL}$ *100)

(5 g)

Ácidos grasos libres $=4,73 \mathrm{mg} \mathrm{KOH}$

gr

Partiendo de este valor ácido obtenido para el aceite, y teniendo en cuenta que la literatura indica que si este valor ácido es mayor a $1 \mathrm{mg} \mathrm{KOH/gr} \mathrm{es}$ necesario realizar la neutralización del aceite para disminuir su acidez. 


\section{Índice de peróxidos:}

La determinación de peróxidos se realizó según la norma NTC 236, en la imagen 2.5 se muestra el color de la muestra antes de la titulación y en la imagen 2.6 se muestra el cambio de color que tuvo esta después de titular con tiosulfato de sodio $0,1 \mathrm{~N}$.

igura 11. Color inicial-Índice de peróxidos

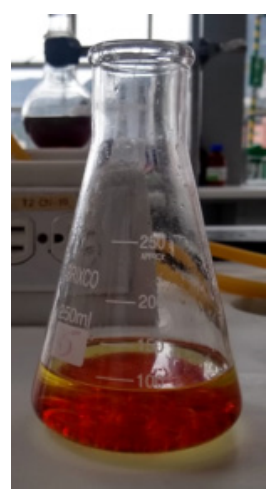

Figura 12. Color final-Índice de peróxidos

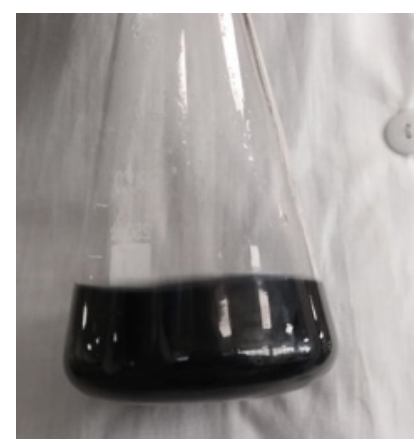

A continuación, se presenta la ecuación con la cual se calculará el valor de peróxido o VP en mili equivalentes de oxígeno por kilogramo:

Donde:

$$
V P=\frac{\left(V-V_{0}\right) * c_{\text {thio }} * F * 1000}{m}
$$

V: Volumen en ml de la solución de tiosulfato de sodio utilizado para la determinación.

V0: Volumen en ml de la solución normal de tiosulfato de sodio utilizado para la muestra control.

F: Es el factor de la solución de tiosulfato de sodio $0.01 \mathrm{~N}$.

cthio: Concentración en moles por litro de la solución de tiosulfato de sodio. 


\section{Tratamiento del aceite:}

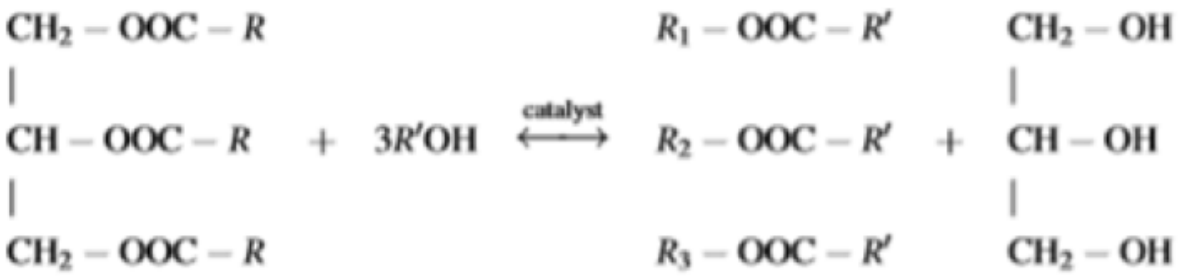

Triglycerides

Alcohol

Esters

Glycerin

\section{Tomado de: (Production of biodiesel from acid oil using sulfuric acid as catalyst: kinetics study)}

Usando la relación estequiométrica de la reacción mostrada anteriormente y tomando una muestra de 100 gr del aceite, se calculó la cantidad de metanol y ácido sulfúrico necesarios para llevar a cabo la reacción, así:

$$
\begin{aligned}
& 100 \mathrm{~g} \text { de aceite } * \frac{1 \mathrm{~mol} \text { de aceite }}{870 \mathrm{~g} \text { de aceite }} * \frac{6 \mathrm{~mol} \text { de metanol }}{1 \mathrm{~mol} \text { de aceite }} * \frac{32,04 \mathrm{~g} \text { de metanol }}{1 \mathrm{~mol} \text { de metanol }} \\
& =22,09 \mathrm{~g} \text { de metanol }
\end{aligned}
$$

Tomando 1 \% p.p del ácido sulfúrico con respecto al peso del aceite:

$$
1 \mathrm{~g} \mathrm{de}_{2} \mathrm{SO}_{4}=\frac{1 \mathrm{ml} \mathrm{de} \mathrm{H}_{2} \mathrm{SO}_{4}}{1,84 \mathrm{~g} \mathrm{de}_{2} \mathrm{SO}_{4}}=0,54 \mathrm{mlde} \mathrm{H}_{2} \mathrm{SO}_{4}
$$


Teniendo en cuenta los cálculos mostrados anteriormente, en la reacción de esterificación (neutralización del aceite) se realizó la mezcla de 27,27 $\mathrm{ml}$ de metanol con 0,54 ml de ácido sulfúrico (catalizador) para llevar a cabo la reacción.

Proceso de esterificación: En la figura 13 se muestra el montaje utilizado para llevar a cabo la neutralización del aceite.

Figura 13. Montaje esterificación del aceite.

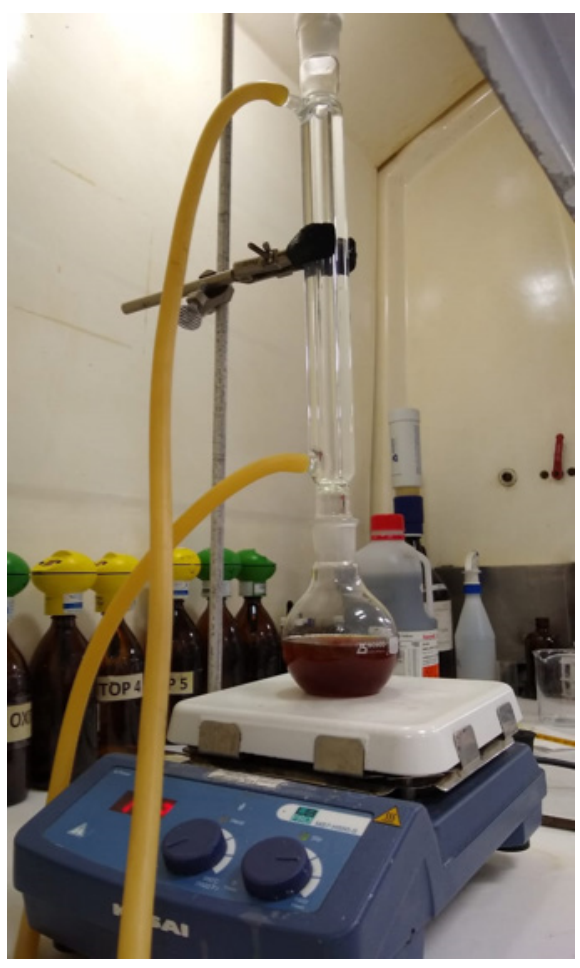

La reacción de esterificación se dejó a temperatura constante (aprox. $60^{\circ} \mathrm{C}$ ) y con agitación constante durante ocho (8) horas. Además, como se puede observar en la figura 13 se hizo uso de un condensador con el fin de no perder el metanol que se pudiera llegar a evaporar, teniendo en cuenta que la reacción se realizó a una temperatura cercana al punto de ebullición de este.

Proceso de producción del biodiesel: Para realizar la neutralización del ácido sulfúrico, y así mismo agregando un exceso de 1 gramo de hidróxido de sodio para que tome lugar como catalizador en la reacción de transesterificación, se tiene: 


\title{
$\mathrm{H}_{2} \mathrm{SO}_{4}+2 \mathrm{NaOH} \leftrightharpoons 2 \mathrm{H}_{2} \mathrm{O}+\mathrm{Na}_{2} \mathrm{SO}_{4}$
}

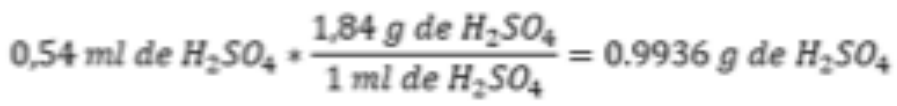 \\ $0,9936 \mathrm{~g}$ de $\mathrm{H}_{2} \mathrm{SO}_{4} * \frac{1 \mathrm{~mol} \mathrm{de}_{2} \mathrm{SO}_{4}}{98,079 \mathrm{~g} \mathrm{de}_{2} \mathrm{SO}_{4}} \cdot \frac{2 \mathrm{~mol} \text { de } \mathrm{NaOH}}{1 \mathrm{~mol} \mathrm{de}_{2} \mathrm{SO}_{4}} \cdot \frac{40 \mathrm{~g} \text { de } \mathrm{NaOH}}{1 \mathrm{~mol} \text { de } \mathrm{NaOH}}$ \\ $=0,81 \mathrm{~g}$ de $\mathrm{NaOH}$
}

Fuente. Elaboración propia

Según los cálculos mostrados, la reacción de transesterificación se realizó con la mezcla de 22,27 ml de metanol y 1,81 g de hidróxido de sodio.

Proceso de transesterificación: Teniendo el aceite a una temperatura controlada y constante de $60^{\circ} \mathrm{C}$, se le agrega la mezcla metanol/ $\mathrm{NaOH}$. Para esta reacción se utilizó el mismo montaje de la esterificación, esta se dejó con calentamiento y agitación constante como se muestra en la imagen 4.1 durante 2 horas.

Figura 14. Reacción de transesterificación.

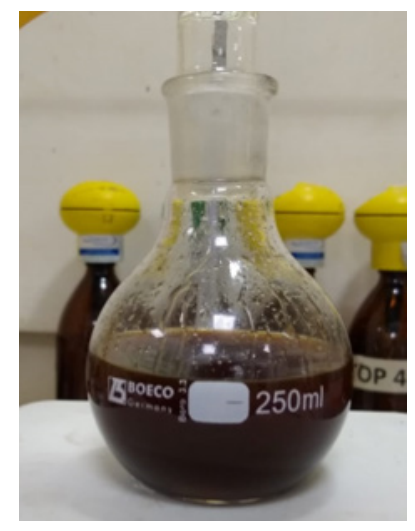

En la figura anterior se puede observar como el metanol se estaba condensando en el balón de fondo plano, lo que indica que se estaba llevando a cabo adecuadamente la reacción de transesterificación para así obtener un buen rendimiento en los productos. 


\section{Separación biodiesel-glicerina.}

Decantación: Después de que el biodiesel se enfriara este se pasó a un embudo de decantación; pasados 15 minutos se pudieron apreciar las dos fases obtenidas, biodiesel-glicerina. Como se muestra en la figura 15 podemos observar que la glicerina queda en la parte de abajo del embudo, mientras el biodiesel por su densidad queda suspendido en la parte superior de este.

Figura 15. Embudo de decantación (Biodiesel-glicerina)

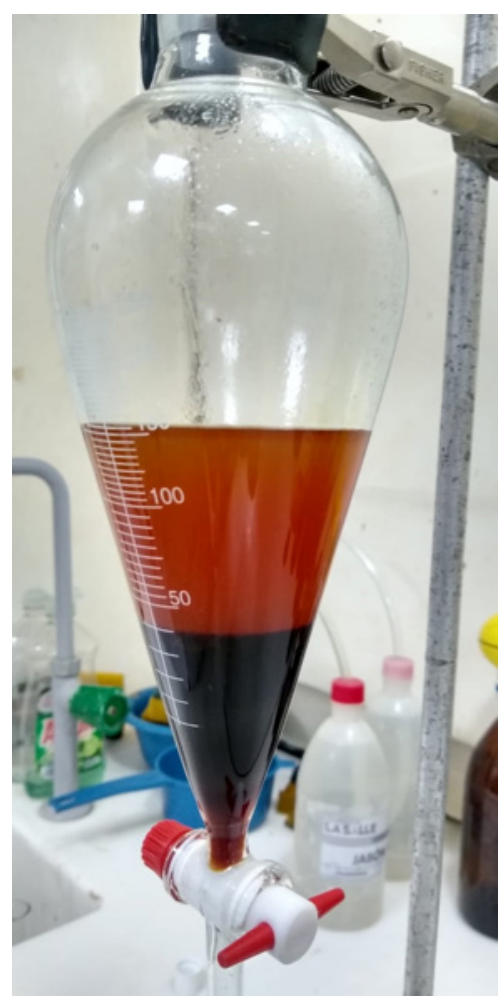

Esta decantación se dejó durante de un día para asegurar la completa separación de estos fluidos. Además, sabiendo que la glicerina se solidifica a temperaturas menores o iguales a $25^{\circ} \mathrm{C}$, fue necesario realizar un baño maría del embudo de decantación para separar el biodiésel y la glicerina. 


\section{Neutralización y lavado.}

Neutralización del catalizador básico: Teniendo en cuenta que se agrega $1 \mathrm{~g}$ de hidróxido de sodio como catalizador para la reacción de transesterificación, es necesario realizar la neutralización de este con ácido sulfúrico.

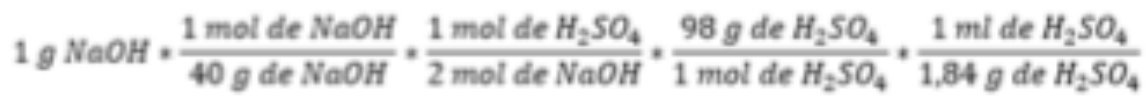

$$
\begin{aligned}
& =0,66 \mathrm{ml} \text { de } \mathrm{H}_{2} \mathrm{SO}_{4}
\end{aligned}
$$

Para esto se agregaron 0,66 ml de ácido sulfúrico al biodiesel y se mezclaron durante 10 minutos.

Lavado del biodiesel: Por cada $10 \mathrm{ml}$ de biodiésel se usaron $50 \mathrm{ml}$ de agua destilada, esta mezcla se agitó durante 10 minutos y después se llevó a un embudo de decantación como se muestra en la imagen 6.1.

Figura 16. Embudo de decantación (Biodiesel-agua)

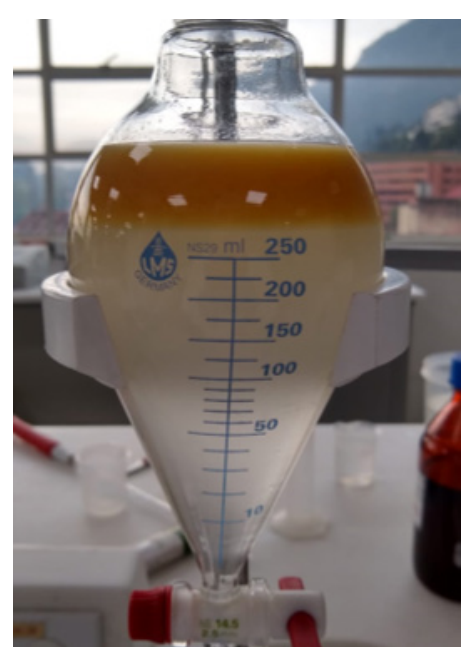


En la figura 16 se observa como el agua queda de un color un turbio después de ser mezclada con el biodiesel, esto sucedió porque se pudieron haber producido jabones en el proceso y estos hacen que el biodiesel se solubiliza en el agua.

\section{Secado.}

Secado del biodiésel: Después de tener el biodiésel lavado, este se lleva a una mufla a $105^{\circ} \mathrm{C}$ para eliminar el agua que haya podido quedar en este, como se muestra en las figuras 17 y 18 este tenía aproximadamente un 10\% de contenido de agua.

Figura 17. Peso inicial del biodiésel

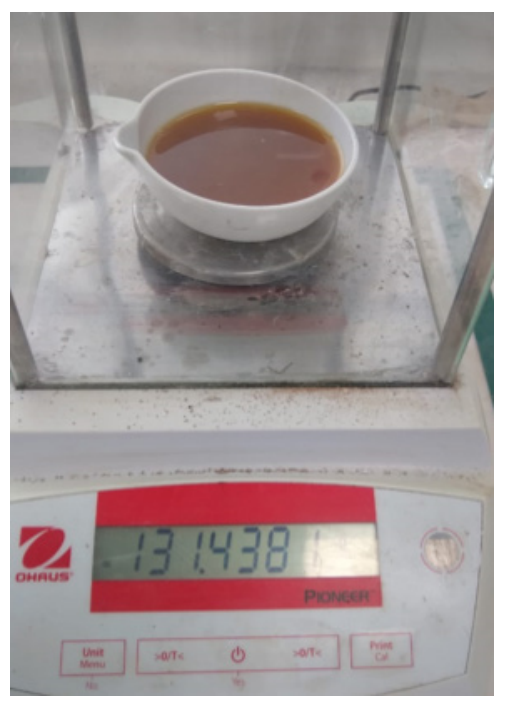

Figura 18. Peso final.

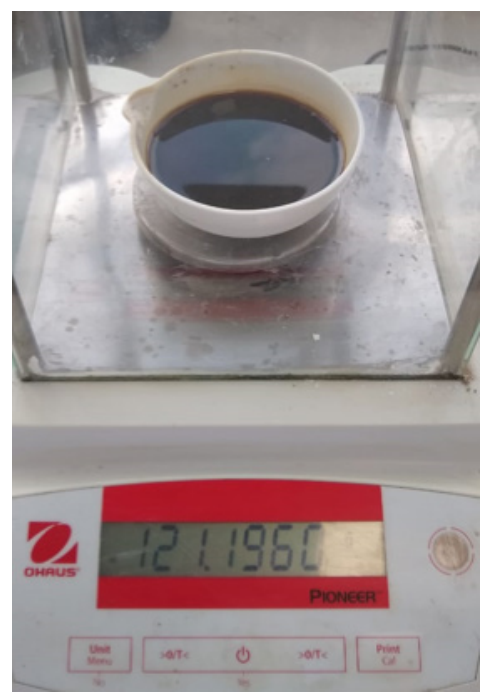

\section{Caracterización del biodiesel.}

Se realizó el mismo procedimiento empleado para la caracterización del aceite, así mismo en el índice de acidez y peróxidos se llevaron a cabo los mismos cálculos, obteniendo los resultados mostrados en la tabla 5. 
Tabla 5. Caracterización del biodiesel

\begin{tabular}{|c|c|c|}
\hline Tipo de análisis / Fluido & Biodisel & Aceite \\
\hline Densidad (g/mL) & 0,88 & 0,918 \\
\hline $\begin{array}{c}\text { Ácidos grasos libres } \\
\text { (mg KOH/gr muestras) }\end{array}$ & 0,21 & 4,73 \\
\hline $\begin{array}{c}\text { Indice de peroxidos } \\
\left(m g_{2} / \mathbf{k g}\right)\end{array}$ & 2,16 & 4,74 \\
\hline Viscosidad (MPa *S) & 29,26 & 131,9 \\
\hline
\end{tabular}

Como se observa en la figura 18 el biodiésel obtenido tiene un color café oscuro y presentaba una baja viscosidad. Inicialmente, al realizar el análisis de la densidad se obtiene una densidad promedio de $0,88 \mathrm{~g} / \mathrm{mL}$, siguiendo con la caracterización se realizó el índice de acidez, el cual se redujo significativamente obteniendo un valor de 0,21 mg de $\mathrm{KOH} / \mathrm{gr}$ mientras el valor del índice de acidez del aceite usado tenía un valor de 4,73 mg de $\mathrm{KOH} / \mathrm{gr}$, teniendo en cuenta lo que indica la norma astm para biodiesel este valor ácido está dentro del valor requerido su uso en la industria (máximo 0,5 mg de KOH/gr).

La última prueba de la caracterización que cobra importancia para determinar si el biodiesel obtenido se puede comercializar, es la prueba de viscosidad donde se obtiene un valor de 29,26 mPa*s, a diferencia del aceite que tuvo un valor de 131,9 mPa*S, con esta viscosidad dinámica para el biodiesel se evidencia que este biocombustible puede ser llevado al mercado nacional ya que cumple con los lineamientos de la norma astm. 


\section{Conclusiones}

De la caracterización fisicoquímica del aceite de cocina usado se obtuvo un valor ácido de 4,73 mg de KOH/gr, lo que indicó que realizar la etapa primaria de esterificación es fundamental para disminuir este índice de acidez, mejorar el rendimiento de la reacción y no favorecer la saponificación durante el proceso.

En la transesterificación básica se evidenció que el rendimiento del biodiesel fue de aproximadamente del 70\%. Aun así, en el lavado se perdió un $20 \%$ del biodiésel debido a que se generaron algunos jabones en la reacción volviendo el biodiésel soluble en el agua.

En la caracterización fisicoquímica del biodiésel, fueron importantes las determinaciones de la viscosidad y el índice de acidez teniendo unos valores de 29,26 mPa*s y 0,21 mg de KOH/gr, respectivamente. Estos valores se encuentran dentro de los rangos requeridos dados por la norma astm de biodiesel, determinando que el biodiesel producido a partir de aceite de cocina usado se puede llevar al mercado nacional.

Para realizar una escala industrial de proceso se requieren referencias de artículos de investigación en base a las bio-refinerías de biodiesel a partir de aceite usado de cocina, aun así, los balances realizados se hicieron en base al procedimiento de laboratorio y hay que tener en cuenta que es necesario realizar la cuantificación de cada uno de los subproductos que se generan a lo largo de todo el proceso para tener todos los flujos definidos.

Por último, habiendo realizado una revisión bibliográfica para llevar a cabo todo el proceso de producción de biodiésel, este proyecto aporta a la generación de conocimiento en esta área de la industria y aún más en la etapa primaria de esterificación que fue en la cual se tuvieron más inconvenientes para encontrar información acerca del proceso. 


\section{Recomendaciones}

- Para hacer de la filtración un proceso más rápido, se debe calentar el aceite a una temperatura de aproximadamente $40{ }^{\circ} \mathrm{C}$.

- En el momento de hacer la mezcla del metanol con el hidróxido de sodio, es indispensable hacerlo en un recipiente cerrado ya que el hidróxido de sodio es poco soluble en el metanol y al final se evapora el alcohol. Para ayudar con la dilución, calentar la mezcla.

- Tener precaución en la mezcla del metanol con el ácido sulfúrico, ya que es una reacción altamente exotérmica.

- Se debe tener precaución a la hora de hacer el índice de peróxidos, debido a que al realizar la titulación esta tiene una reacción demasiado rápida con muy poca cantidad de tiosulfato.

- Teniendo en cuenta el alto valor ácido original del aceite se recomienda considerar su uso en la producción de jabones.

- Se recomienda también desarrollar por separado las etapas de neutralización del ácido sulfúrico y la transesterificación en medio alcalino para obtener los sulfatos y el glicerol por separado.

- Se planea llevar el proceso a escala industrial, realizando un estudio de mercado y llevando a cabo el balance de materia. 


\section{ANEXOS}

NTC 236

- Pesar 5 g de muestra homogeneizada en un Erlenmeyer de $250 \mathrm{~mL}$ con tapa esmerilada.

- Añadir $30 \mathrm{~mL}$ de una solución de ácido acético: cloroformo (3:2) y agitar hasta que se disuelva completamente.

- Añadir $0.5 \mathrm{~mL}$ de solución saturada de KI (o bien 2 g) tapar y agitar durante un minuto en oscuridad.

- Luego de esto, añadir $30 \mathrm{~mL}$ de agua destilada. Proceder a valorar con tiosulfato de sodio $0.01 \mathrm{~N}$, hasta desaparición parcial o total del color amarillo (Es necesario trabajar con un microbureta).

- Nuevamente añadir $30 \mathrm{~mL}$ de agua destilada y adicionar $0.5 \mathrm{~mL}$ de solución de almidón al 1\%. Continuar valoración agitando constantemente hasta desaparición del color azul.

- Hacer un blanco con los reactivos utilizados.

A continuación, se presenta la ecuación con la cual se calculará el valor de peróxido o VP en miliequivalentes de oxígeno por kilogramo:

$$
V P=\frac{\left(V-V_{0}\right) * c_{\text {thio }} * F * 1000}{m}
$$

Donde:

- $\quad$ : Volumen en $\mathrm{ml}$ de la solución de tiosulfato de sodio utilizado para la determinación.

- Vo: Volumen en ml de la solución normal de tiosulfato de sodio utilizado para la muestra control. 
- F: Es el factor de la solución de tiosulfato de sodio 0.01N.

- cthio: Concentración en moles por litro de la solución de tiosulfato de sodio.

- m: Masa en gramos de la muestra analizada.

El factor F se determina a partir de la ecuación:

$$
F=\frac{m_{K^{\prime O} O_{3}} * V_{1} * 6 * 1000 * w_{K I O_{3}}}{M_{K_{1} O_{3}} * V_{2} * V_{3} * c_{\text {thio }} * 100}
$$

Donde:

- mKIO3: masa en gramos de yodato de potasio.

- 6: masa equivalente para el título (1 mol KIO3 03 mol I2)

- V1: volumen de la solución de yodato de potasio empleado para la determinación del título (5 o 10 ml).

- V2: volumen total en ml (250 o 500 ml) de la solución de yodato de potasio.

- V3: Volumen en ml de la solución de tiosulfato $0.01 \mathrm{~N}$ utilizado en la determinación

- wKIO3: pureza del yodato de potasio en g/100g.

- MKIO3: Masa molecular del yodato de potasio (214 g/mol).

- cthio: Concentración de la solución normal de tiosulfato de sodio en moles por litro $(0.01 \mathrm{~mol} / \mathrm{L})$

Valor ácido NTC 218:

- Pesar 5-10 g de muestra bien mezclada en un Erlenmeyer de 250 mL. 
- Agregar 50-100 mL de mezcla de alcohol-éter.

- Mezclar y luego se procede a agregar $0.1 \mathrm{~mL}$ de solución de fenolftaleína.

- Luego de esto se titula con $\mathrm{KOH}$ alcohólico 0,1 N en movimiento constante hasta que aparezca una coloración rosa tenue permanente y persiste por un tiempo mayor a 15 segundos.

Según la NTC 218, el valor ácido, WAV, se reporta como se presenta a continuación:

- Para valores entre 0 hasta 1 se reportan dos cifras decimales;

- Para valores entre 1 hasta 100 se reporta una cifra decimal;

- Para valores > 100 como número entero

El valor ácido, expresado se calculará con la ecuación:

\% Ácidos grasos libres $=\underline{56,1 \times \mathrm{V} \times \mathrm{N} \times 100}$

P

Donde:

$V=$ volumen $(\mathrm{ml})$ de $\mathrm{NaOH}$ consumidos

$\mathrm{N}=$ Normalidad del $\mathrm{NaOH}$

$\mathrm{P}=$ peso (g) de aceite 


\section{Referencias bibliográficas}

[1] Y Zhang, M.A Dubé, D,D McLean and $M$ Kates (2003). Biodiesel production from waste cooking oil: 1. Process design and technological assessment. 1st ed. [ebook] Ottawa, Canada: ELSEVIER. Available at: https://www.sciencedirect. com/science/article/pii/S0960852403000403 [Accessed 15 Aug. 2019].

[2]Siegel Moecke, E., Feller, R., Hélia Alice dos Santos, Machado, M., Vieria Cubas and et, all. (2016). Biodiesel production from waste cooking oil for use as fuel in artisanal fishing boats: Integrating environmental, economic and social aspects. 1st ed. [ebook] Brazil: ELSEVIER. Available at: https://www.sciencedirect.com/science/article/pii/S0959652616306461 [Accessed 28 Sep. 2019].

[3]Glisic, S. and Orlovic, A. (2014). Review of biodiesel synthesis from waste oil under elevated pressure and temperature: Phase equilibrium, reaction kinetics, process design and techno-economic study. 1st ed. [ebook] Belgrade, Serbia: ELSEVIER. Available at: https://www.sciencedirect. com/science/article/pii/S1364032113008174 [Accessed 3 Oct. 2019].
[4]Consejo Nacional de Política Económica y Social (2008). Documento CONPES 3510 de 2008. 1st ed. [ebook] Bogotá D.C. Available at: https://www.fenalce.org/archivos/conpesbiocombustibles.pdf [Accessed 10 Oct. 2019].

[5] [Internet]. fedebicombustibles.com. 2019 [cited 18 September 2019]. Available from: https://www.fedebiocombustibles.com/ nota-web-id-1231.htm

[6]Producción de biocombustibles, afectada por importaciones más baratas desde EE. UU. - Valora Analitik [Internet]. Valora Analitik. 2019 [cited 18 September 2019]. Available from: https://www.valoraanalitik. com/2018/12/20/produccion-colombiana-de-biocombustibles-afectada-por-importaciones-mas-baratas-desde-ee-uu/

[7] Ronderos and Cardenas (2016). ESTUDIO DE MERCADO - ACEITES Y GRASAS. [ebook] Bogotá. Available at: https://sistemas.mre.gov. br/kitweb/datafiles/Bogota/es-es/file/Estudio\%20de\%20Mercado\%20-\%20Aceites\%20 y\%20Grasas.pdf [Accessed 6 Oct. 2019]. 